\title{
McDONALD OBSERVATORY ARCHIVE OF OPTICAL LINEAR POLARIZATION MEASUREMENTS
}

\author{
Beverley J. WiLls ${ }^{1,2}$, D. WiLls ${ }^{1}$, AND M. BREger ${ }^{1,3}$ \\ ${ }^{1}$ University of Texas Astronomy Department C1400, 1 University Station, Austin, TX 78712, USA; bev@astro.as.utexas.edu \\ ${ }^{2}$ University of Texas McDonald Observatory C1401, 1 University Station, Austin, TX 78712, USA \\ ${ }^{3}$ Institut für Astronomie, University of Vienna, Türkenschanzstrasse 17, 1180 Vienna, Austria \\ Received 2010 October 27; accepted 2011 March 5; published 2011 April 29
}

\begin{abstract}
We present 990 previously unpublished optical linear polarization measurements of quasars, active galactic nuclei, and some stars observed for interstellar polarization. The observations, covering the period 1981-2000, were made with McDonald Observatory's $2.1 \mathrm{~m}$ Struve reflector and the Breger photopolarimeter.
\end{abstract}

Key words: catalogs - polarization - quasars: general

Online-only material: machine-readable table

\section{INTRODUCTION}

Polarimetry provides a unique probe of astrophysical conditions, through measurement of linear and circular polarization, providing insights unavailable from the vast majority of observations made in total light. The main physical mechanisms are dichroic transmission through aligned grains, synchrotron emission from extragalactic jets, and scattering by dust or ionized gas. Polarization by dichroic transmission gives information on the direction and degree of alignment of grains in the interstellar medium of our and other galaxies. Polarized synchrotron emission allows us to deduce the alignment of magnetic fields in radio quasar jets. Polarization of higher energy photons gives us clues to field alignments, and shocks within parsecs of the quasar nuclei, especially in the blazars-quasars whose optical spectra are often dominated by rapid, large amplitude variations in synchrotron emission. Scattering by dusty or ionized gas is important for its role in revealing faint halos of scattered light surrounding dust-obscured active galactic nuclei (AGNs) and quasars (Antonucci 1993). Here we present results of 970 observations, including 960 optical linear polarization measurements of 295 quasars and AGNs, as well as some stars, close on the sky plane, observed to estimate interstellar polarization. The observations, covering the period from 1981 to 2000, were made with the Breger photopolarimeter mounted on the Struve $2.1 \mathrm{~m}$ telescope of the University of Texas' McDonald Observatory. While many other results have been published (e.g., Breger 1979; Breger \& Wheeler 1980; Wills et al. 1980; Breger \& Cochran 1982; Hsu \& Breger 1982; Breger 1986; Berdyugin et al. 1992; Wills et al. 1992a, 1992b; Hines \& Wills 1993; Andersson \& Wannier 1995), the results of smaller, less complete, programs have not. Some unpublished observations were part of undergraduate projects made to investigate, e.g., the interstellar polarization contributions to the low polarization measured toward lobe-dominant quasars. Many observations were part of a program to follow up on the "duty cycle" of variable quasars (Wills et al. 1992a), that is, what patterns might be discerned in the fraction of time blazars may be "turned on" (practically, often defined as when the degree of polarization $p>3 \%)$. Some programs were incomplete as a result of the vagaries of weather. A significant fraction of these unpublished measurements were from observations made through clouds or in poor seeing (e.g., we followed the polarization variability of a number of relatively bright blazars because measurements of the degree of polarization and position angle (P.A.) for these are little affected by cloud and poor seeing). The data presented here are useful for the above-mentioned studies, and papers based on these data are in preparation (e.g., Caldwell et al. 2011).

\section{OBSERVATIONS}

These observations were made with the Breger photopolarimeter mounted at the $f / 13$ Cassegrain focus of the $2.1 \mathrm{~m}$ Struve reflector of McDonald Observatory. The instrument is described by Breger (1979). Briefly, light passes through a nonmetallic aperture, then through an analyzer-a Glan-Air prism rotating at $12-24 \mathrm{~Hz}$. The transmitted light ( $E$ vector) passes through a Lyot depolarizer, a filter wheel, a Fabry lens, thence to the cathode of a photomultiplier. The depolarizer is important to eliminate any polarization sensitivity of succeeding elements-filters, Fabry lens, and cathode. The rapid rotation ensures accurate observations of polarization, even in conditions of variable atmospheric transmission. While the accuracy of the present observations of faint quasars is photon limited (as confirmed by repeated observations), the instrument was capable of polarization precision at the $0.01 \%$ level on bright stars. Data taking was synchronized with the rotation of the Glan prism.

Two photomultiplier tubes were used. All observations before 1987, and for the 1987 November and 1988 April runs, used EMI 9658 tubes, with S20 cathodes and extended red response. For the 1987 February and March runs, a Hamamtsu R943-02 tube with Ga-As photocathode was used, again with extended red response. Otherwise, for 1988 July and later, we used a Hamamatsu R943-02 with a specially selected cathode for excellent extended red response. The filter bandpasses were chosen to be suitable for measurement of the wavelength dependence of polarization. $U, B$, and $V$ are similar to Johnson system filters, with effective wavelengths of approximately $0.35 \mu \mathrm{m}(U), 0.44 \mu \mathrm{m}(B)$, and $0.54 \mu \mathrm{m}(V)$. Interference filters were chosen to more accurately define the wavelengths at $R(0.65 \mu \mathrm{m})$ and $I(0.75 \mu \mathrm{m})$ bands (see Table 1 of Breger 1979). For faint quasars, it is often more efficient to use filters of broader bandwidth than the standard UBVRI; thus we have used a copper sulfate filter for the blue, and a Schott RG630 glass filter (usually $3 \mathrm{~mm}$ thick) for the red. We have determined the effective wavelengths for these broader bands using the spectral response of the R943-02 photomultiplier provided by the 
Table 1

McDonald Observatory Broadband Linear Polarization Archive

\begin{tabular}{|c|c|c|c|c|c|c|c|c|c|c|c|c|c|}
\hline \multirow{2}{*}{$\begin{array}{l}\text { Name } \\
(1950)\end{array}$} & \multirow[t]{2}{*}{ Other Name } & \multirow[t]{2}{*}{ ISP } & \multicolumn{3}{|c|}{ UT Date } & \multirow[t]{2}{*}{ MJD } & \multicolumn{2}{|c|}{$p \%$} & \multirow[t]{2}{*}{$\sigma_{p} \%$} & \multirow[t]{2}{*}{ P.A. } & \multirow[t]{2}{*}{ Filt } & \multirow[t]{2}{*}{ Notes } & \multirow[t]{2}{*}{ Program } \\
\hline & & & year & month & day & & & & & & & & \\
\hline $0004+0147$ & LBQS & $\ldots$ & 1989 & Sep & 26 & 47795.2917 & 1 & 13 & 0.4 & 118.0 & $\ldots$ & BALQ, ap $5^{\prime \prime} .3$ & $\ldots$ \\
\hline $0007+106$ & III Zw 2 & $\ldots$ & 1987 & Nov & 24 & 47123.2917 & 1 & 83 & 0.63 & $\ldots$ & $\ldots$ & Thick cloud, unreliable & $\ldots$ \\
\hline $0010-0012$ & LBQS & $\ldots$ & 1989 & Sep & 26 & 47795.2917 & 1 & 7 & 0.77 & 116.0 & $\ldots$ & BALQ, ap 5..2 & $\ldots$ \\
\hline $0014+813$ & S5 & $\ldots$ & 1988 & Oct & 15 & 47449.2917 & 0 & 99 & 0.27 & 136.7 & $\ldots$ & $\ldots$ & Antonucci disk project \\
\hline $0014+813$ & S5 & $\ldots$ & 1989 & Jan & 12 & 47538.2917 & 0 & 91 & 0.27 & 95.0 & $\ldots$ & $\ldots$ & $\ldots$ \\
\hline $0019+0107$ & LBQS & $\ldots$ & 1989 & Sep & 28 & 47797.2917 & 0 & 44 & 0.31 & 21.0 & $\ldots$ & BALQ, ap $5 .{ }^{\prime \prime 2}$ & $\ldots$ \\
\hline $0020-0154$ & LBQS & $\ldots$ & 1989 & Sep & 27 & 47796.2917 & 0 & 34 & 0.9 & 172.0 & $\ldots$ & BALQ, ap $5^{\prime \prime} 2$ & $\ldots$ \\
\hline $0021-0213$ & LBQS & $\ldots$ & 1989 & Sep & 28 & 47797.2917 & 0 & 95 & 0.94 & 27.0 & $\ldots$ & BALQ, ap $5^{\prime \prime} .2$ & $\ldots$ \\
\hline $0025-0151$ & LBQS & $\ldots$ & 1989 & Sep & 26 & 47795.2917 & 1 & 02 & 0.45 & 99.0 & $\ldots$ & BALQ, ap $5 .{ }^{\prime \prime 2}$ & $\ldots$ \\
\hline $0029+0017$ & LBQS & $\ldots$ & 1989 & Sep & 27 & 47796.2917 & 1 & 2 & 1.1 & 26.0 & $\ldots$ & BALQ, ap 5".2 & $\ldots$ \\
\hline $0048-097$ & PKS & $\ldots$ & 1988 & Oct & 18 & 47452.2917 & 18 & 13 & 1.08 & 129.5 & $U$ & $\ldots$ & $\ldots$ \\
\hline $0048-097$ & PKS & $\ldots$ & 1988 & Oct & 18 & 47452.2917 & 16 & 83 & 0.75 & 131.7 & $I$ & $\ldots$ & $\ldots$ \\
\hline
\end{tabular}

(This table is available in its entirety in a machine-readable form in the online journal. A portion is shown here for guidance regarding its form and content.)

manufacturer, atmospheric transmission through a model atmosphere applied to McDonald Observatory (E. S. Barker 1980, private communication), spectral measurements obtained at McDonald Observatory of typical atmospheric atomic and molecular absorption features, and the broadband filter transmissions. We have assumed photon-counting appropriate to the mode used for the polarimeter photomultiplier, together with a canonical $F_{\lambda} \propto \lambda^{-1}$ quasar continuum:

$$
\lambda_{\text {eff }}=\left(\int \lambda\left(\lambda F_{\lambda}\right) R_{\lambda} d(\lambda)\right) /\left(\int\left(\lambda F_{\lambda}\right) R_{\lambda} d(\lambda)\right),
$$

where $R_{\lambda}$ is the combined spectral atmospheric absorption, filter transmission, and phototube response. For no filter, $\mathrm{CuSO}_{4}$, and RG630 filters, the estimated effective wavelengths are $5475 \AA$, $4200 \AA$, and $7350 \AA$. These apply to $p \%$, assumed constant across the band, which is often true for blazars and quasars that are dominated by a synchrotron continuum.

Observations were generally made in units of integration time of $200 \mathrm{~s}$, determined to be optimal given the phototube noise characteristics. We alternated between object-centered and offset sky observations, in sky-object-object-sky patterns, the pattern and total integration times depending on object count rate. Nearly all observations were made through a circular aperture of projected diameter 7".4. Most observations were made with the Glan prism rotation rate of $12 \mathrm{~Hz}$. Detector dead-time was determined each run, and corrections applied automatically to the count rate and polarization, $p$. The sine curves are displayed and the object, sky and sky-subtracted count rates and polarizations are calculated and updated in real time. The analyzer efficiency was confirmed to be $100 \%$ by inserting a "calibration polaroid" into the beam. The small instrumental polarization $(p \lesssim 0.05 \%)$ and zero point of the P.A. were determined nightly via unpolarized and polarized standard stars (Hsu \& Breger 1982), referred to the systematic polarization P.A. frame defined by Serkowski (1974). The P.A. zero point was checked throughout the night by observing bright stars through the calibration polaroid.

\section{THE McDONALD OBSERVATORY ARCHIVE}

Table 1 presents previously unpublished polarization measurements of quasars and a few lower-luminosity AGNs, together with some stars observed to check for interstellar polarization. Column 1 gives the IAU-style name formed from the B1950 coordinates. A "Q" preceding most of these will be recognized by the SIMBAD database and the NASA/IPAC Extragalactic Database (NED). Column 2 gives another name or survey designation, to aid in identifying the object. At the end of entries for a given quasar are given the measurements of nearby bright stars that may be suitable for constraining the Galactic interstellar polarization toward the quasar. These measurements are indicated by "ISP" in Column 3. Columns 4-6 give the UT year, month, and day of the observation. Column 7 gives the corresponding Modified Julian Date, MJD, which is here defined MJD = JD-2400000.5. Where there is only a single measurement of a source on one night, we assume UT $7 \mathrm{hr}$ to correct approximately to the average MJD for that night at McDonald Observatory. Where there are several observations on a given night, the MJD is given to an accuracy of a few seconds. The percentage polarization $p \%$ and its rms uncertainty are given in Columns 7 and 8 . We have not applied any bias correction to $p \%$ (as described by Wardle \& Kronberg 1974); it seems simpler to allow for direct computations in normalized Stokes parameters $q$ and $u$, for example, when combining polarization vectors. The uncertainty, $\sigma_{p} \%$, which is always dominated by photon statistics, is determined from the count rates on "object plus sky" and sky. The P.A., in the equatorial frame, measured, as is usual, from north through east, is given in Column 9. A good estimate of the statistical uncertainties in P.A. is given by $28.65 \times \sigma_{p \%} / p \%$. The uncertainty in defining the zero point of P.A. is about $1^{\circ}$. The filter used for the observation is given in Column 10-no entry means no filter. Sometimes, where spectral dependence of polarization has been measured, we have also combined results from different filters, as indicated. Column 11 gives other source names, especially where there may be ambiguity in the short 1950 IAU name information, or as a way of identifying the stars chosen for ISP measurements. We also indicate where non-standard apertures were used, although this information may not be complete. Finally, Column 12 gives the sample, project name, or principal investigator. Further details and references are given at the end of the table. We particularly note we give only sufficient results to define the polarization behavior of blazars intensively monitored by DW (OJ $287=0851+202$, PKS 0829+046, $0219+428=3 \mathrm{C} 66 \mathrm{~A})$. The complete data set for these will be published later.

Interstellar polarization can sometimes be a significant source of uncertainty, both in $p \%$ and P.A. Even near the north 
Galactic pole and south Galactic pole, polarization of distant stars can be $\approx 0.2 \%$ (Berdyugin et al. 2004). The suitability of the polarization measurements for determining interstellar polarization toward the quasar needs to be assessed. Ideally, we would choose intrinsically unpolarized stars very close to the quasar sight line but whose light traverses a great distance through our Galaxy and we therefore attempted to observe luminous blue stars of spectral type B or earlier, close on the sky to the quasar. Sometimes, there were no suitable stars within $1^{\circ}$, which presents a problem because the interstellar medium can be clumpy on small and large scales. Another approach is to search recent catalogs of stellar polarization. Heiles (2000) carefully compiled available data up to that time, and at high Galactic latitudes the Berdyugin et al. (2004, and references therein) catalogs are a valuable resource. From the original polarization maps by Mathewson \& Ford (1970) it is seen that there appear some regions of tangled interstellar magnetic field direction, but, fortunately, others where P.A. (and to a lesser extent, $p \%$ ) appear to be fairly uniform. More recent maps, for high Galactic latitudes $|b|>70^{\circ}$ (Berdyugin et al. 2004), are useful in indicating large regions of aligned P.A. In the present paper, we do not investigate interstellar polarization further.

We are grateful to the helpful and knowledgeable staff at McDonald Observatory who have maintained the photopolarimeter and detectors (E. Dutchover, D. R. Doss), software (D. Crook, M. Ward), and willingly helped with nighttime equipment emergencies (E. Green, T. Brown, G. Grubb). We thank our colleagues, especially Dean C. Hines, who have contributed to our programs. This research has made use of (1) NASA's Astrophysics Data System Service, (2) the NASA/IPAC Extragalactic Database (NED), which is operated by the Jet Propulsion Laboratory, California Institute of Technology, under contract with the National Aeronautics and Space Administration, and (3) the SIMBAD database (CDS, Strasbourg, France), the VizieR cata- log access tool (Ochsenbein et al. 2000), and the Aladin sky atlas tool (Bonnarel et al. 2000). The measurements presented in this paper were obtained as part of research supported by the National Science Foundation under grant numbers AST 79-01182, AST-8215477, AST 8714937, and AST 02-06261.

Facility: Struve

\section{REFERENCES}

Andersson, B.-G., \& Wannier, P. G. 1995, ApJ, 443, L49

Antonucci, R. 1993, ARA\&A, 31, 473

Berdyugin, A., Breger, M., \& Polosukhina, N. 1992, A\&A, 255, 149

Berdyugin, A., Piirola, V., \& Teerikorpi, P. 2004, A\&A, 424, 873

Boller, T., Bertoldi, F., Dennefeld, M., \& Voges, W. 1998, A\&AS, 129, 87

Bonnarel, F., et al. 2000, A\&AS, 143, 33

Breger, M. 1979, ApJ, 233, 97

Breger, M. 1986, ApJ, 309, 311

Breger, M., \& Cochran, W. D. 1982, Icarus, 49, 120

Breger, M., \& Wheeler, J. C. 1980, PASP, 92, 514

Caldwell, C., Wills, B., Wills, D., Aller, H., \& Aller, M. 2011, BAAS, 43, 2011

Grupe, D., Komossa, S., Leighly, K. M., \& Page, K. L. 2010, ApJS, 187, 64

Grupe, D., Wills, B. J., Wills, D., \& Beuermann, K. 1998, A\&A, 333, 827

Heiles, C. 2000, AJ, 119, 923

Hines, D. C., \& Wills, B. J. 1993, ApJ, 415, 82

Hsu, J.-C., \& Breger, M. 1982, ApJ, 262, 732

Kühr, H. 1977, A\&AS, 29, 139

Mathewson, D. S., \& Ford, V. L. 1970, Mem. R. Astron. Soc., 74, 139

Meisenheimer, K., \& Röser, H.-J. 1983, A\&AS, 51, 41

Ochsenbein, F., Bauer, P., \& Marcourt, J. 2000, A\&AS, 143, 230

Serkowski, K. 1974, in IAU Colloq. 23, Planets, Stars \& Nebulae Studied with Photopolarimetry, ed. T. Gherels (Tucson, AZ: Univ. of Arizona), 135

Tapia, S., Craine, E. R., Gearhart, M. R., Pacht, E., \& Kraus, J. 1977, ApJ, 215, L71

Wardle, J. F. C., \& Kronberg, P. P. 1974, ApJ, 194, 249

Wills, B. J., Wills, D., Breger, M., Antonucci, R. R. J., \& Barvainis, R. 1992a, ApJ, 398, 454

Wills, B. J., Wills, D., Evans, N. J., II, Natta, A., Thompson, K. L., Breger, M., \& Sitko, M. L. 1992b, ApJ, 400, 96

Wills, D., Wills, B. J., Breger, M., \& Hsu, J.-C. 1980, AJ, 85, 1555

Wilson, A. S., Ward, M. J., Axon, D. J., Elvis, M., \& Meurs, E. J. A. 1979, MNRAS, 187, 109 